\title{
Distributed Full Coverage WSN Protocol Based on Energy Self-Aware
}

\author{
Li Shuqiang $^{1}$ \\ ${ }^{1}$ College of Agricultural Engineering, Henan University of Science and \\ Technology, Luoyang, 471003, China \\ ${ }^{1}$ E-mail: dr_lsq@163.com
}

\begin{abstract}
With maximizing the coverage time by designing effective algorithm being the core problem for large-scale network, a kind of distributed energy-aware and coverage-aware full-coverage WSN routing protocol (DEAOP) is proposed in this paper. Protocol put forward in this paper adopts the cluster technology. Firstly, divide the sensor nodes into different clusters, with each cluster selecting the cluster head $(\mathrm{CH})$ according to residual energy of the nodes and overlapping degree of the coverage area, and other nodes serve as member nodes of the cluster. Secondly, establish the main data transmission line constituted by cluster head $\mathrm{CH}$. The transmitted data, together with the cluster head $\mathrm{CH}$, is applied to calculate the cost of neighbor cluster head, and nodes with higher cost can be taken as the forwarding node for next group of data. The cost function contains relevant information on residual energy of the nodes and path loss; hence, the higher the cost is, the greater the residual energy and the lower the path loss will be. In this case, nodes with less residual energy will not become invalid prematurely due to being selected as the cluster head $\mathrm{CH}$, thus avoiding the resulting short service life of network and balancing the network energy consumption. Finally, simulate the protocol and analyze its performance in network service life, coverage rate and energy consumption. The simulation results indicate that protocol proposed in this paper can lower the energy consumption and prolong the network service life, thus improving the coverage rate.
\end{abstract}

Keywords: Network protocol; Energy-aware; WSNs; Routing; Coverage rate; Sensor node

\section{Introduction}

As a network mechanism in which data transmission is carried out by wireless communication, Wireless Sensor Network (WSN) is constituted by a large number of sensor nodes, with each node distributed in the monitoring area. Various sensor nodes in the network can collect, calculate and analyze the information on relevant objects in the network coverage area. In general, sensor nodes distributed randomly are deployed in the area where monitoring is required; however, since the density of these nodes is relatively high, it also brings the problem of high redundancy. To lower the impact of sensor network abnormality on normal operation of the network, scholars have put forward many coping algorithms. For instance, Rodrigo.R [3] et al. put forward a kind of algorithm for sensor network abnormality searching based on a refreshing mechanism for regional interaction characteristics. In this algorithm, the mode of periodic characteristic sampling was adopted to refresh the regional characteristics, thus quickly extracting the aggressive behavior of abnormal nodes. However, this kind of searching algorithm can only achieve recursion of the past abnormal aggressive behavior, and frequent updating of nodes can easily lead to serious misjudgment, thus lowering the adaptability of the algorithm. Li.H.T [4] et al. proposed a kind of algorithm for sensor network node searching based on an isolating detection mechanism for abnormal behavior. This algorithm adopted the 
partitioned mode, and once abnormality was found in an area, the isolating mechanism would be quickly started up, thus reducing the harm caused by such abnormality to the greatest extent. In this algorithm, however, no adequate consideration has been taken into the node density distribution; when the nodes are distributed in high density, a large number of normal nodes will be divided into the isolated area, thus leading to a largescale node paralysis in the network. Ning.H.S [5] et al. put forward a kind of algorithm for sensor network abnormality discrimination based on a data watermarking discrimination mechanism. In this algorithm, irrevocable temporary watermark was added to the data message, thus lowering the possibility of abnormal nodes sneaking into disorderly. Nevertheless, this algorithm requires that all the data messages sent out by nodes should be added with watermark, and when the network data flow is relatively large, complexity of the algorithm will be increased, with a rising trend in data transmission delay in the network.

\section{Sensor Network Model}

In consideration of the fact that the sensor network, during deployment, often adopts the sensor based on wireless data exchange mode as the network node, and these nodes complete the networking [6] by adopting the random distribution mode, once an abnormal situation occurs, abnormal information perception can be achieved by the self-provided transceiver antenna and the wireless perception mode. For convenience sake, assume that nodes of the entire sensor network are distributed within the $N \times N$ rectangular region [7]. With regard to sensor network node $i$, provided that its perceptual position within the above-mentioned rectangular region is $\left(x_{i}, y_{i}\right)$, as is shown in Figure 1; when any abnormality of the node is detected, the central node $j$ can be set as the detection node (coordinate as $(x, y))$,

$P(i, j)=1,|i, j|<R$

Where, $P(i, j)$ is the detection probability, $|i, j|$ represents the physical distance between $i$ and $j$, while $R$ represents the optimal perceptual radius of central node $j$ :

$|i, j|=\sqrt{\left|x_{i}-x\right|^{2}+\left|y_{i}-y\right|^{2}}$

According to Model (1) and Model (2), a judgment can be made in whether the abnormality in node $i$ can be detected. If after being processed by Model (1), detection probability of all the network nodes is 1 , it indicates that the sensor network is in a perceptible state. Otherwise, the partitioned mode needs to be adopted, and different detection nodes need to be set in different partitions to ensure that entire network is in a perceptible state.

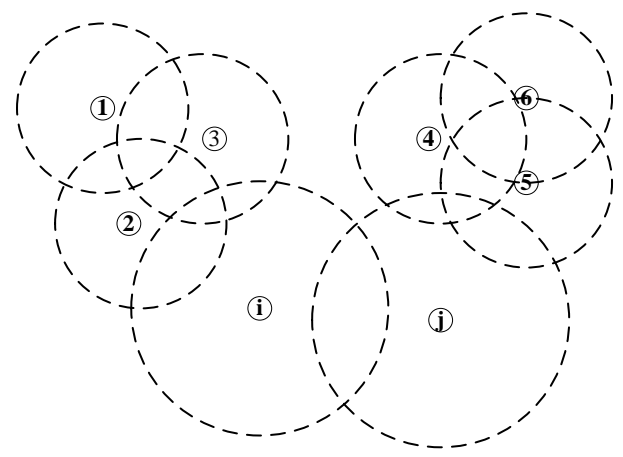

Figure 1. Schematic Diagram of Sensor Network Node Coverage 


\section{Full-coverage Route of Capacity-aware Network}

In WSN, failure of sensor nodes will lead to coverage holes and degrade performance of the entire network. If a sensor node fails, other nodes should move to make up the blind sensing area caused by failure of such node, thus maintaining performance of the entire network [8-9]. In the DEAOP algorithm, therefore, each sensor node, on a regular basis, sends Heartbeat Message to the neighbor nodes to inform them of their working state. If the neighbor nodes fail to receive the Heartbeat Message within a certain period, it indicates that the node may be invalid. Where a sensor node receives the signal from other nodes, whether the node fails or not can be judged according to formula (1). When the signal RSS is less than the threshold value $N_{t h}$, the node can be judged as invalid node. Once an invalid node is detected, other nodes shall move in a proper way. As is shown in Figure 2, failure of sensor node F leads to coverage hole, and in this case, other nodes need to move appropriately.

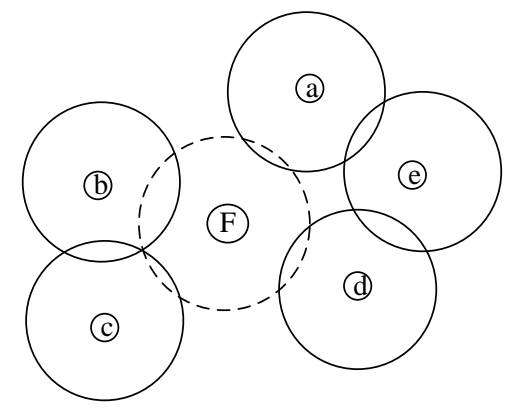

\section{Figure 2. Schematic Diagram of Invalid Node}

Where an invalid node is detected, it is required to move a node from the neighbor nodes, for the purpose of minimizing the coverage-free area. As is shown in Figure 4, invalid node $\mathrm{F}$ has many neighbor nodes, and there is a chance that movement of a neighbor node can minimize the coverage-free area.

Therefore, the DEAOP algorithm should be adopted to select the appropriate mobile node. In the RCH-FL algorithm, neighbor nodes will firstly select the most appropriate mobile node [10] by adopting the FLS2 (Type-2 Fuzzy logic System), and then FLS will, in accordance with the node energy $\mathrm{E}$, distance $\mathrm{d}$ from the hole area and coverage redundancy $\xi_{i}$, select the optimal mobile node. Finally, the target location for the mobile node will be determined by adopting FLS1. Model for whole process of the RCH-FL algorithm is shown in Figure 3.

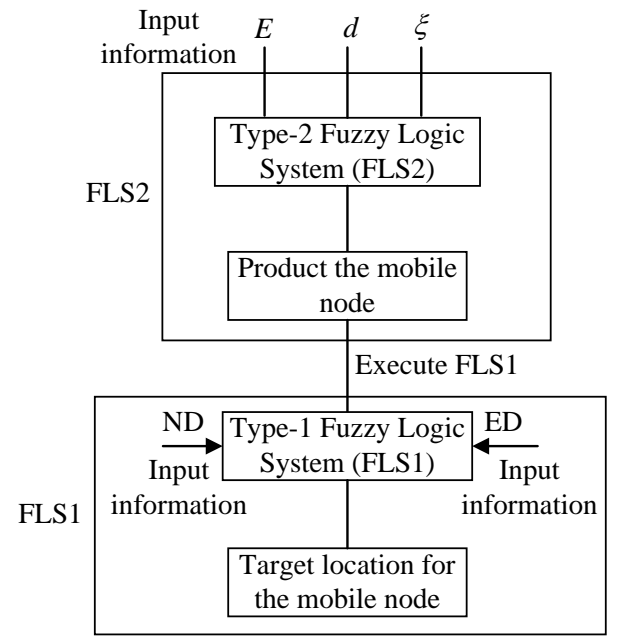

Figure 3. FL-Based RCH-FL Algorithm Model 
FLS2 is constituted by three input and one output language variables, and the output variable represents the possibility of the sensor node being selected:

$T(\lambda)=\{$ Very high, high, Medium, low, verylow $\}$

Subjection degree of the energy $\mathrm{E}$, distance $\mathrm{d}$ from the hole area and coverage redundancy $\xi_{i}$ is shown in Figure 4.

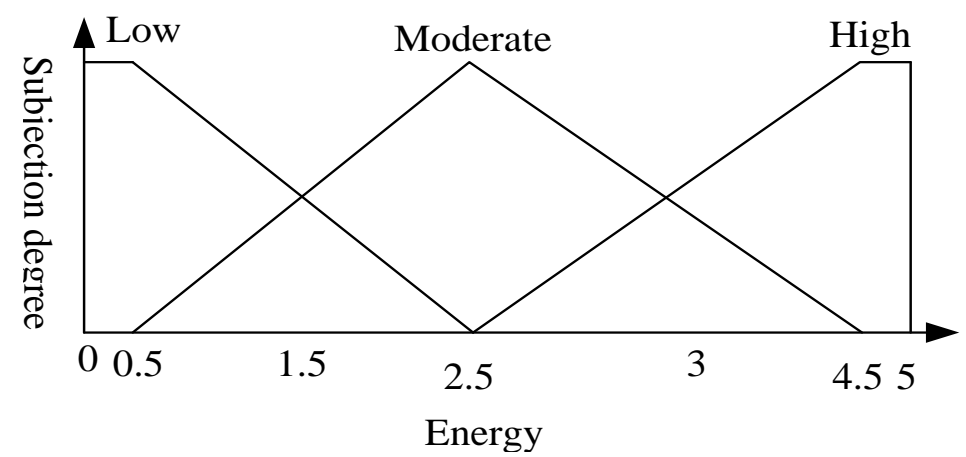

(a)

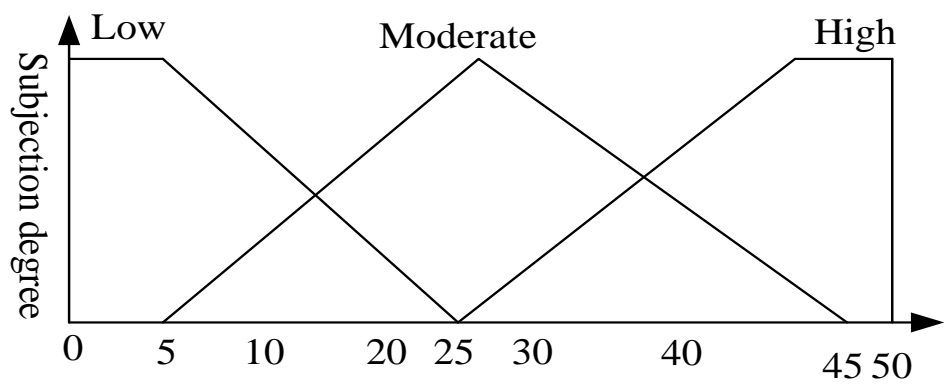

Distance

(b)

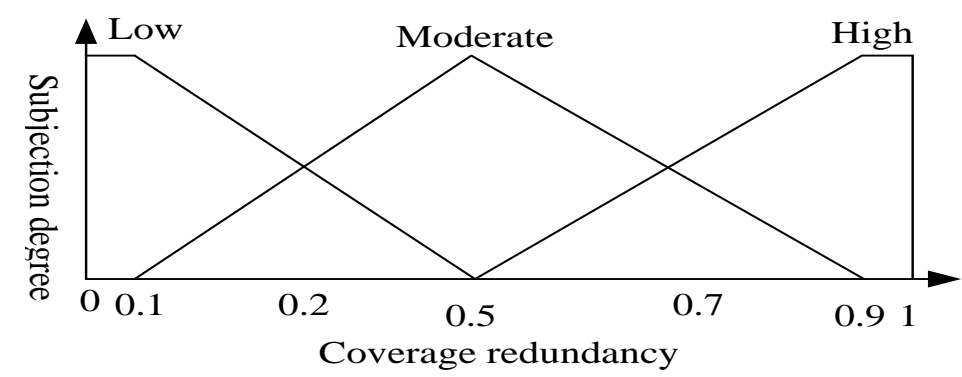

(c)

Figure 4. Subjection Degree of the Three Inputs

Sequence the neighbor nodes by virtue of the three information items, and the sequencing basis is shown in Table 3. As is shown in Table 1, the smaller the serial number is, the greater the probability of being selected will be. After selecting the node, corresponding movement will be conducted; then the selected node will move with the FLS1 algorithm as the basis, as is shown in Figure 5. Figure 5 sets out to describe the whole process of hole repairing by the mobile node. Aimed at failure of node F, node E, according to the FLS2 algorithm, is selected as the mobile node to repair the coverage hole [11-13] caused by failure of node F. 
Table 1. Rule Base of FLS2

\begin{tabular}{|c|c|c|c|c|}
\multirow{2}{*}{ rule } & \multicolumn{5}{|c|}{ Input variables } \\
\cline { 2 - 5 } & Energy & Distance & $\begin{array}{c}\text { Coverage } \\
\text { redundanc }\end{array}$ & $\begin{array}{c}\text { Probability of } \\
\text { being selected }\end{array}$ \\
\hline 1 & High & High & High & VHigh \\
\hline 2 & High & High & Medium & VHigh \\
\hline 3 & High & High & Low & High \\
\hline 4 & High & Medium & High & High \\
\hline 5 & High & Medium & Medium & High \\
\hline 6 & High & Medium & Low & Medium \\
\hline 7 & High & Low & High & Medium \\
\hline 8 & High & Low & Medium & Medium \\
\hline 9 & High & Low & Low & Low \\
\hline 10 & Medium & High & High & Medium \\
\hline 11 & Medium & High & High & Low \\
\hline 12 & Medium & High & Low & Low \\
\hline 13 & Medium & Medium & High & Medium \\
\hline 14 & Medium & Medium & Medium & Medium \\
\hline 15 & Medium & Medium & Low & Low \\
\hline 16 & Medium & Low & High & Medium \\
\hline 17 & Medium & Low & Medium & Low \\
\hline 18 & Medium & Low & Low & VLow \\
\hline 19 & Low & High & High & High \\
\hline 20 & Low & High & Medium & Medium \\
\hline 21 & Low & High & Low & Low \\
\hline 22 & Low & Medium & High & Medium \\
\hline 23 & Low & Medium & Medium & Low \\
\hline 24 & Low & Medium & Low & VLow \\
\hline 25 & Low & Low & High & Low \\
\hline 26 & Low & Low & Medium & VLow \\
\hline 27 & Low & Low & Low & VLow \\
\hline
\end{tabular}

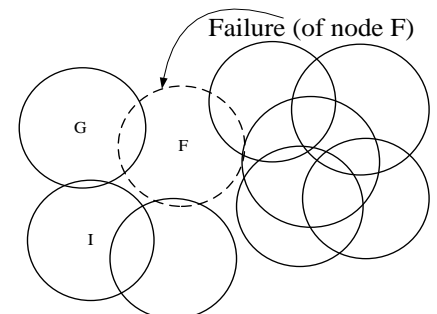

(a) Coverage hole appears

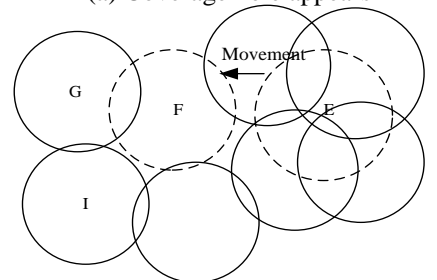

(b)Select node E as the mobile node

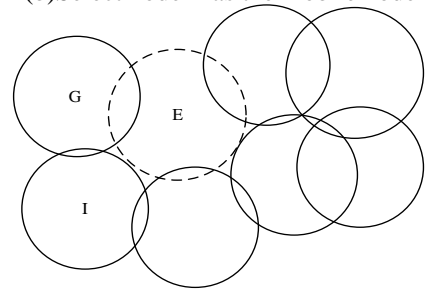

(c)After hole repairing

Figure 5. Schematic Diagram for the Process of Hole Repairing by Mobile Node 


\section{Communication Module Design}

\subsection{Realization of Server-side Communication Module}

For server side, on the one hand, it needs to receive environmental data frequently uploaded by environment collection equipment; on the other hand, it needs to handle data request of mobile phone client side. Faced with the two kinds of different communication requests, server-side adopts two kinds of different communication methods. (1) Adopt non-blocking communication method based on Socket for communication among environment collection equipment. (2) Adopt Http Client method based on HTPP for communication among mobile phone client sides.

Socket communication method is a blocking-type. After server side monitors Socket connection of client side, it will launch a new thread to conduct data interaction; each Thread will only dispose one Socket connection; only after client side breaks connection automatically, server side will release resource. In the environment of Internet of Things, a large number of sensors equipment will connect with system; adopting blocking-type communication will only lead to reduction of server performance. Java NIO non-blocking communication after JDK1.4 (Java Development Kit) can meet communication requirement of high concurrency. Java NIO realizes non-blocking communication by three important components of Buffer, Channel and Selector. Herein, Buffer is used for store all kinds of format data temporarily. Channel is used to read and write data that is sent from client side to server side. Selector is used to manage Channel and dispatch a Thread to dispose many Channels.

System server side firstly sets non-blocking passage by realization type Server Socket Channel of Channel; creates Socket monitoring and binds Channel in Selector manager by register method. When client side connects with server, Selector manager will select a Socket Channel example object treatment and interaction with client side by Selector manager when client side connects with server. Release the Socket Channel after closure of interaction.

There are methods of Socket and Http Client in network communication of Android system. Client side can better integrate with Spring MVC framework in server by adopting Http Client method. Mobile phone client side can send POST request to server by Http Client. Server will encapsulate requested data into JSON format and return it to mobile phone client side.

\subsection{Realization of Client-side Equipment Management Module}

To reduce use complexity of system, system will add environment collection equipment by adopting the method of scanning 2-dimensional code. 2-dimensional code is a kind of new information storage and transmission technology. Express binary data in the method of black and white pattern according to specific coding rule and in the method of black and white matrix pattern. Inclusive information [11] can be obtained after equipment scanning it. At present, the most extensive 2-dimensional code is QR code (Quick Response Code). This kind of 2-dimensional code can put in 1817 Chinese characters to the maximum. Initialization parameters of several sensors of a set of equipment can be stored by the method of 2-dimensional code. Users can obtain initialization information of equipment after scanning the 2-dimensional code. The equipment will be added to system.

In system, increase of equipment is realized by adding Device Activity type. Thirdparty library file qrcode. jar will be introduced to recognize 2-dimensional code. This type firstly invokes mobile phone camera to capture image of 2-dimensional code and stores it as Buffered mage object; transmit Buffered Image to decode method of qrcode type as parameters. After analysis of 2-dimensional code by this method, return inclusive information in2-dimensional code in the method of character string. After system obtains 
initialization information of equipment, users are allowed to modify initialization information according to actual condition. Users can add new equipment to system after kicking storage button.

\section{Experiment and Analysis}

\subsection{Network Service life and Energy Consumption}

Firstly, analyze energy consumption and invalid cluster-head number in scheme of the paper. Result is shown in Figure 6. In the condition of same round, energy consumption put forward in DEAOP scheme of the paper is less than CPCP-ea and EEUC scheme. It is mainly because that CPCP-ea protocol does not consider invalid condition of cluster head and energy utilization rate is low. When cluster head is invalid, it still transmits data to cluster head when cluster head is invalid. Therefore, energy is wasted. However, EEUC protocol is superior to CPCP-ea protocol. It is because that EEUC protocol considers invalid condition of cluster head $\mathrm{CH}$. Besides, Figure 6 also describes invalid number of cluster head $\mathrm{CH}$ of several protocols. It is shown in data that invalid cluster head $\mathrm{CHs}$ proposed in protocol of the paper is obviously less than CPCP-ea and EEUC.
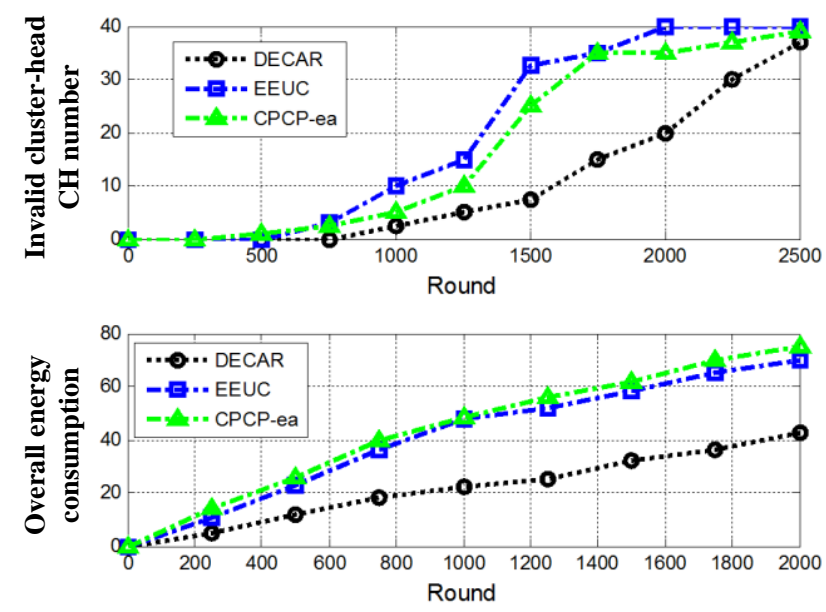

Figure 6. Energy Consumption

Table 2. Relationship between Energy Consumption and Running Round Number

\begin{tabular}{ccccccccc}
\hline \multirow{2}{*}{ Test } & \multicolumn{2}{c}{ Energy consumption $50 \%$} & & \multicolumn{3}{c}{ Running 1000 rounds } \\
\cline { 2 - 3 } \cline { 6 - 8 } & CPCP-ea & EEUC & DECAR & & CPCP-ea & EEUC & DECAR \\
\hline 1 & 475 & 476 & 1038 & & 48.89 & 47.35 & 23.49 \\
2 & 477 & 474 & 1088 & & 48.43 & 46.95 & 21.87 \\
3 & 475 & 476 & 1109 & & 48.48 & 47.76 & 21.23 \\
4 & 479 & 484 & 1092 & & 48.60 & 47.76 & 21.23 \\
5 & 475 & 484 & 1123 & & 48.99 & 47.01 & 20.24 \\
6 & 478 & 480 & 1058 & & 48.69 & 47.30 & 22.89 \\
7 & 474 & 473 & 1076 & & 48.94 & 47.48 & 22.71 \\
8 & 482 & 472 & 1060 & & 48.66 & 47.83 & 22.61 \\
9 & 469 & 483 & 1097 & & 48.81 & 47.30 & 21.48 \\
10 & 475 & 478 & 1116 & & 48.60 & 47.38 & 21.03 \\
\hline
\end{tabular}

Table 2 lists part of experience data. From Table 2, it can be known that protocol put forward in the paper has run for 1082 rounds when energy is consumed nearly $50 \%$. CPCP-ea and EEUC has respectively run for 477 rounds and 478 rounds. It can be known 
from energy consumption data that energy consumption utilization rate of protocol in the paper has been promoted for $127.0 \%$ and $126.6 \%$ compared with that of CPCP-ea and EEUC. For example, when it runs for 1000 rounds, protocol put forward in the paper only consumes 22.0J energy. However, CPCP-ea protocol and EEUC protocol respectively has consumed $48.6 \mathrm{~J}$ and $47.5 \mathrm{~J}$.

Table 3. Active Nodes Number and Rounds Number of the First Invalid Nodes

\begin{tabular}{ccccccccc}
\hline \multirow{2}{*}{ Test } & \multicolumn{3}{c}{$\begin{array}{c}\text { Active nodes number } \\
\text { (running for 2000 rounds) }\end{array}$} & & \multicolumn{3}{c}{ Network service life } \\
\cline { 2 - 4 } \cline { 6 - 8 } & CPCP-ea & EEUC & DECAR & & CPCP-ea & EEUC & DECAR \\
\hline 1 & 1 & 9 & 25 & & 754 & 736 & 1274 \\
2 & 1 & 6 & 15 & & 755 & 716 & 1228 \\
3 & 1 & 9 & 19 & & 754 & 739 & 1265 \\
4 & 1 & 5 & 18 & & 775 & 758 & 1303 \\
5 & 2 & 6 & 21 & & 783 & 676 & 1231 \\
6 & 1 & 10 & 21 & & 761 & 731 & 1328 \\
7 & 1 & 5 & 16 & & 782 & 696 & 1148 \\
8 & 1 & 8 & 25 & & 760 & 759 & 1329 \\
9 & 1 & 4 & 22 & & 764 & 723 & 1320 \\
10 & 1 & 9 & 21 & & 772 & 769 & 856 \\
\hline
\end{tabular}

Table 3 lists two items of data in experience: 1) after running for 2000 rounds, active nodes number; 2) total running rounds of the first invalid nodes, namely network service life. It can be known from Table 3 that occurrence time of protocol put forward in the paper on the first invalid nodes has improved for $66.9 \%$ compared with CPCP-ea protocol and for $71.9 \%$ compared with EEUC protocol. These performance promotions owe to residual energy of protocol utilization node energy and selective cluster head of cover overlapping ratio put forward in the paper.

\subsection{Contrast Experiment of Ability Consumption}

The purpose to put forward RCH-FL algorithm is to move position of sensor nodes and to realize the maximal region coverage with the minimal energy consumption. To analyze consumed energy of sensor nodes in the moving process, establish the following scene: sensor nodes distribute randomly for 8 times. After each random deployment, part of sensor nodes will move and reach the same coverage rate. Analyze average energy consumed by sensor nodes in the movement process under the environment. Results are shown in Figure 7.

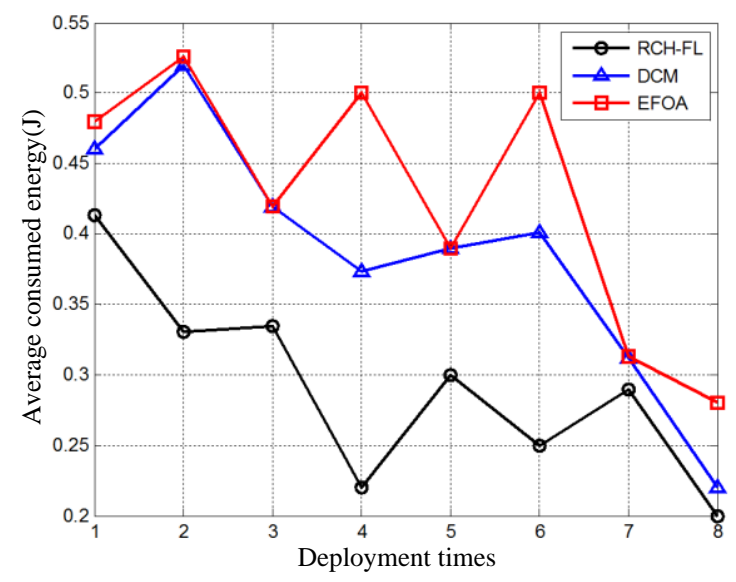

Figure 7. Average Energy Consumption 
It can be known from Figure 7 that average energy consumption of DEAOP algorithm is lower than DCM and EFOA. The reason is that RCH-FL algorithm selects mobile nodes correctly. Mobile nodes move accurately to destination according to local node density and Euclidean distance information. Energy consumption of EFOA is the highest because it adopts random movement strategy, movement blindness exists and energy consumption is increased. Besides, DCM scheme energy utilization rate is lower than RCH-FL algorithm. Because DCM searches movement position by GPS and running GPS and receiving signal all need energy, so energy consumption is increased.

\subsection{Contrast Experiment of Coverage Rate}

Figure 8 describes change curve of coverage rate with the number of sensor nodes; coverage rate will rise with the increase of the number of sensor nodes. Herein, coverage rate of DEAOP algorithm is the highest. Average coverage rate reaches $99.5 \%$; however, DCM is $90 \%$ and EFOA is only $82 \%$. Contrast results of coverage rate are shown as Figure 8.

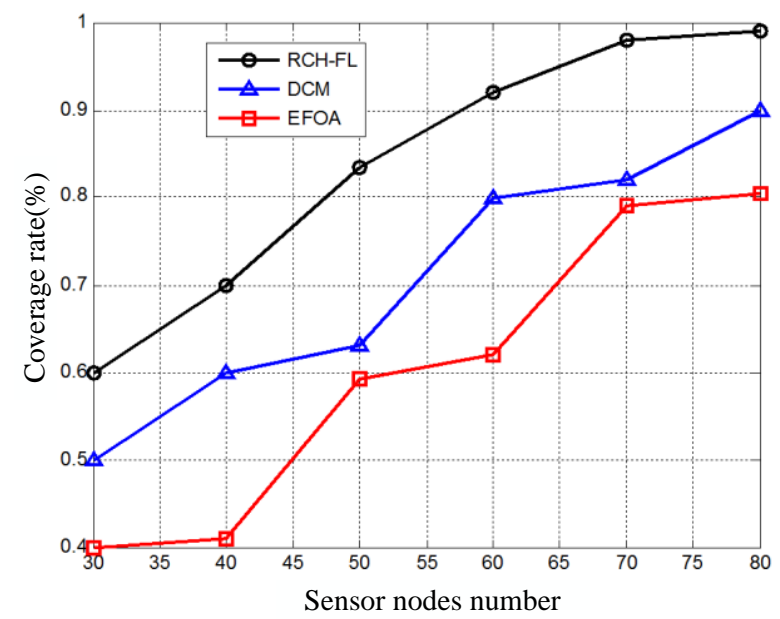

Figure 8.Change Curve of Coverage Rate with Sensor nodes Number

\section{Conclusion}

Because uneven distribution of sensor nodes, exhausted energy and faults, coverage hole always exists in WSNs. On the one hand, coverage hole leads to blind spot in sensor nodes when collecting data; on the other hand, data transmission will break off and therefore, performance of the whole network will be influenced. Therefore, the paper puts forward RCH-FL algorithm based on fuzzy logic coverage hole repair. RCH-FL algorithm quotes the concept of mobile nodes repairing hollow. Select the optimal mobile nodes by making use of fuzzy logic and combining with information of three aspects of energy of sensor nodes, distance from hollow and coverage redundancy. Subsequently, calculate position of mobile target by making use of nodes density and regional average Euclidean distance. Simulated results show that RCH-FL algorithm can repair coverage hole effectively. 


\section{References}

[1] Su T, Wang W, Lv Z. Rapid Delaunay triangulation for randomly distributed point cloud data using adaptive Hilbert curve[J]. Computers \& Graphics, (2016), 54: 65-74.

[2] Gu W, Lv Z, Hao M. Change detection method for remote sensing images based on an improved Markov random field[J]. Multimedia Tools and Applications, (2015): 1-16.

[3] Lv Z, Tek A, Da Silva F. Game on, science-how video game technology may help biologists tackle visualization challenges[J]. PloS one, (2013), 8(3): 57990.

[4] Guo C, Liu X, Jin M. The research on optimization of auto supply chain network robust model under macroeconomic fluctuations[J]. Chaos, Solitons \& Fractals, (2015).

[5] $\mathrm{Li} \mathrm{X}, \mathrm{Lv} \mathrm{Z}, \mathrm{Hu}$ J. XEarth: A 3D GIS Platform for managing massive city information[C]//Computational Intelligence and Virtual Environments for Measurement Systems and Applications (CIVEMSA), 2015 IEEE International Conference on. IEEE, (2015): 1-6.

[6] Yang J, Chen B, Zhou J. A Low-Power and Portable Biomedical Device for Respiratory Monitoring with a Stable Power Source[J]. Sensors, (2015), 15(8): 19618-19632.

[7] Su T, Wang W, Lv Z. Rapid Delaunay triangulation for randomly distributed point cloud data using adaptive Hilbert curve[J]. Computers \& Graphics, (2016), 54: 65-74.

[8] Jinyu Hu, Zhiwei Gao and Weisen Pan. Multiangle Social Network Recommendation Algorithms and Similarity Network Evaluation[J]. Journal of Applied Mathematics, 2013 (2013).

[9] Jinyu Hu and Zhiwei Gao. Modules identification in gene positive networks of hepatocellular carcinoma using Pearson agglomerative method and Pearson cohesion coupling modularity[J]. Journal of Applied Mathematics, 2012 (2012).

[10] Lv Z, Tek A, Da Silva F. Game on, science-how video game technology may help biologists tackle visualization challenges[J]. PloS one, (2013), 8(3): 57990.

[11] Guanxiong Liu, Yishuang Geng, Kaveh Pahlavan, Effects of calibration RFID tags on performance of inertial navigation in indoor environment, 2015 International Conference on Computing, Networking and Communications (ICNC), Feb. (2015).

[12] Jie He, Yishuang Geng, Yadong Wan, Shen Li, Kaveh Pahlavan, A cyber physical test-bed for virtualization of RF access environment for body sensor network, IEEE Sensor Journal, 13(10), 38263836, Oct. (2013).

\section{Authors}

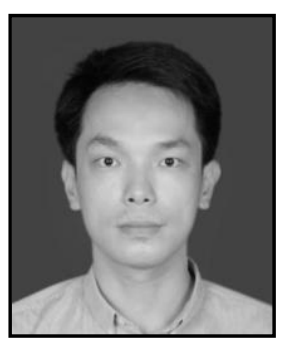

Li Shuqiang, He received Ph.D. in agricultural engineering from China Agricultural University in Beijing, China. He is currently a lecturer in the Henan University of Science and Technology in Luoyang, China. His research interest is mainly in the area of Precision Agricultural, Agricultural Engineering. He has published several research papers in scholarly journals in the above research areas and has participated in several books. 